\title{
Sub-nanosecond Pulse Generation from a Two-section Laser-thyristor: Theoretical Analysis
}

\section{Vyacheslav Golovin ${ }^{1}$, Dmitry Romanovich ${ }^{1,2}$, Olga Soboleva ${ }^{1}$, Aleksandr Podoskin ${ }^{1}$, Polina Gavrina ${ }^{1}$, Vladimir Kapitonov ${ }^{1}$, Dmitry Veselov ${ }^{1}$, Sergey Slipchenko1, and Nikita Pikhtin ${ }^{1}$ \\ ${ }^{1}$ Ioffe Institute, 194021, St. Petersburg, Russia \\ ${ }^{2}$ Saint Petersburg Electrotechnical University, 197376, St. Petersburg, Russia}

\section{Abstract}

We have developed a theoretical model of a two-section laser-thyristor. It is shown that using a $4 \mu \mathrm{m}$ weakly doped p-base can increase blocking voltage up to $50 \mathrm{~V}$, which makes it possible to generate 2 ns 10 A current pulses. It is demonstrated that the proposed device utilizes passive $\mathrm{Q}$ switching to generate high-power short optical

Corresponding Author: Vyacheslav Golovin v.s.golovin@gmail.com

Received: 22 July 2018 Accepted: 9 September 2018 Published: 8 October 2018

Publishing services provided by Knowledge E

(c) Vyacheslav Golovin et al. This article is distributed under the terms of the Creative Commons Attribution License, which permits unrestricted use and redistribution provided that the original author and source are credited.

Selection and Peer-review under the responsibility of the Breakthrough Directions of Scientific Research at MEPhl Conference Committee.

\section{G OPEN ACCESS}

pulses that account for up to $80 \%$ of output power. By picking the optimal passive section length, we have achieved optical pulses of $\sim 30 \mathrm{ps}$ full width at half maximum (FWHM) and $\sim 100 \mathrm{~W}$ peak power.

Keywords: pulsed laser, semiconductor N-p-N-i-P heterostructure, laser-thyristor, dynamic model of pulsed laser

\section{Introduction}

Today, the problem of generating high-power laser pulses of ns and sub-ns duration via semiconductor lasers is of great interest [1-3]. Semiconductor lasers in comparison to other lasers are cheaper, more energy efficient and compact. They are used in different areas such as range finding, LIDAR systems, free space communication and nonlinear frequency conversion. At the moment, the typical way to pump laser diodes is to use external pulsed power supplies or high-power electrical circuits based on bipolar and field-effect transistors. However, the downsides of this approach are the presence of parasitic inductive losses on external circuit elements and a need for coupling with a low-resistance load (laser diode). Also, as the power increases and the pulse duration decreases, such sources become more expensive while their energy efficiency gets lower. The laser-thyristor is a fundamentally new approach for generating laser pulses, which incorporates epitaxial integration of a thyristor current switch (a current pulse generator) and a laser diode within a single heterostructure. This approach leads to a 
minimization of overall size of the device, to an elimination of electrical losses caused by the elements of the power circuit and to an increase of wall-plug efficiency. Thus, it is possible to create compact and cost-efficient pulsed laser sources based on a laserthyristor.

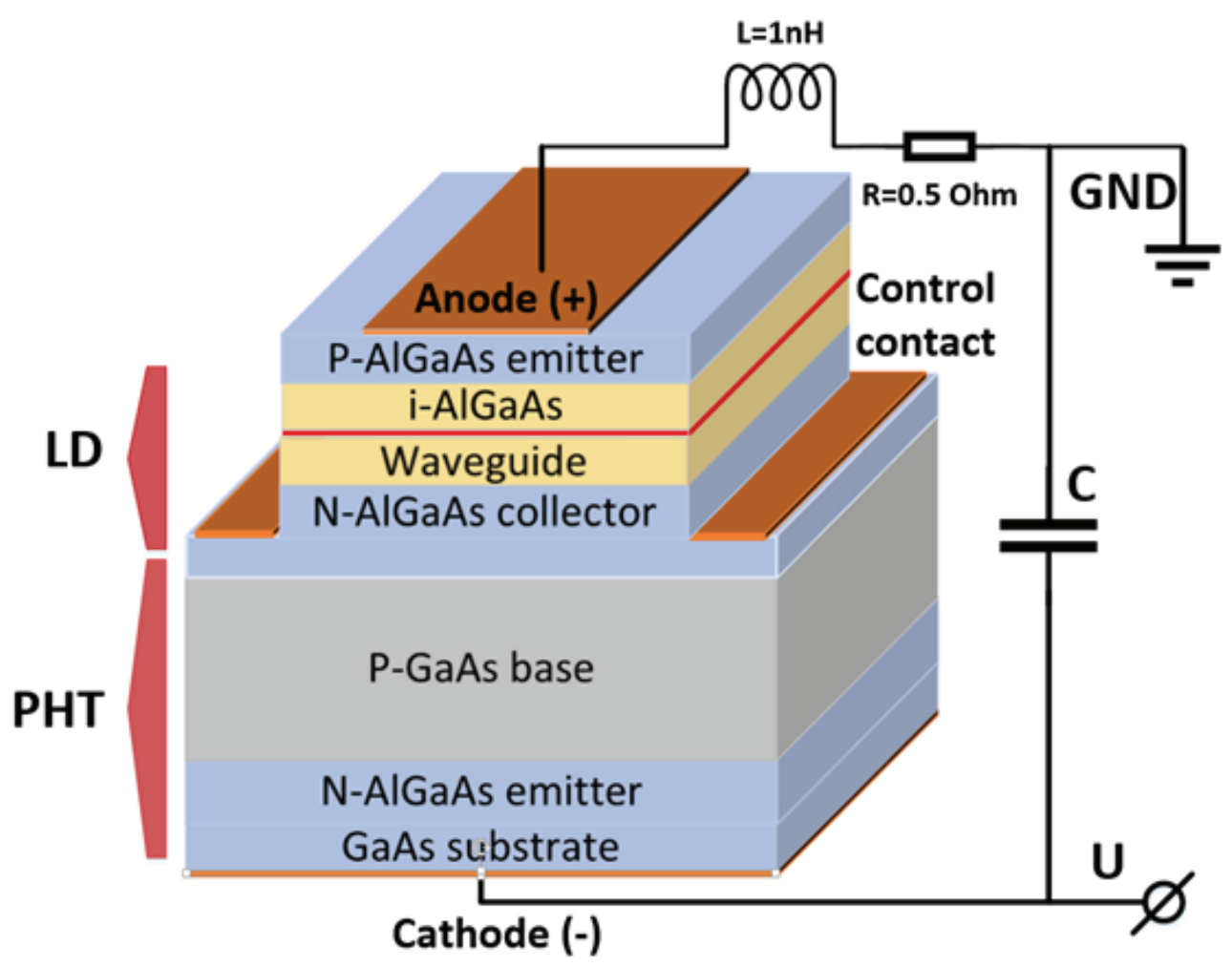

Figure 1: Schematic of a laser-thyristor.

Laser-thyristor structure as well as the corresponding electric circuit is shown in Figure 1 (the inductance of the circuit is represented as a separate element). The laser-thyristor consists of an $\mathrm{N}-\mathrm{p}-\mathrm{N}$ transistor part and a laser $\mathrm{N}-\mathrm{i}-\mathrm{P}$ part. This structure that makes it possible to form two stable states - turned-ON (low resistance) and turned-OFF (high resistance). Unlike classical thyristor structures, the feedback is provided by nonlinear optical feedback [4]. When a control pulse is applied, the current flows through the laser diode. Spontaneous emission is generated in the active region, is being partially absorbed in the base region. Photogenerated carriers activate impact ionization in the reverse-biased collector junction (collector-base). Accumulation of nonequilibrium holes in the base region leads to the turn-ON of a laser-thyristor, the current begins to flow through the structure due to the discharge of an extrinsic capacitor connected with the device in parallel. The current pulse flowing through the heterostructure activates the laser diode. The turn-OFF occurs due to the capacitor discharge, since the current falls below the hold-ON current. 
This approach's feasibility was demonstrated in [5], and in [6] laser pulses of 47 and $8 \mathrm{~W}$ peak optical power were obtained at pulse length of 100 and $10 \mathrm{~ns}$, respectively. However, the most promising direction is generating ns and sub-ns high power. To obtain sub-ns pulses in a laser diode via pumping with current pulses of ns length, several approaches such as gain switching and Q-switching are used $[1,7,8]$. In this article, the authors present the results of modeling lasing dynamics in passive Qswitching mode in a two-section laser-thyristor. A laser-thyristor dynamic model was developed in [9], which made it possible to simulate current and voltage dynamics in laser-thyristor structures. This model has proven to be in a good agreement with experimental results.

In [9], it was shown that a laser-thyristor can be represented as an optically connected couple «laser diode-phototransistor», and the transistor part of the device can be considered separately from the laser diode as a current pulse generator. In this case, feedback between the phototransistor part and the laser part is achieved by the means of the active region spontaneous emission absorption by the base of the phototransistor. In [10], we have shown that it is possible to obtain current pulses generation of $15 \mathrm{~A}$ amplitude and 2 ns duration in laser-thyristor structures, if they have an increased breakdown voltage ( $50 \mathrm{~V}$ ). The aim of presented work is to simulate laser dynamics using the data on the current pulses in the laser-thyristor structures obtained in [10].

\section{Model}

In order to describe laser emission dynamics we used a modified version of a system of rate equations (1-3). The two-section model includes an active section and a passive section acting as a saturable absorber (Figure 2 ).

An additional equation describing dynamics of carrier density in the active region of the passive section has been included the system of rate equations. The last term in equation (2) considers absorption of the active section spontaneous emission by the passive section. Due to this, carriers accumulate in the active region of the passive section, which leads to absorption modulation. In equation (3), which describes photon density dynamics, the two sections are considered separately and gain is determined by the carrier density in the corresponding section.

$$
\frac{d n_{1}}{d t}=\eta_{i} \cdot \frac{I_{1}(t)}{q \cdot V q w_{1}}-\frac{n_{1}}{\tau_{s p}\left(n_{1}\right)}-c \cdot S \cdot \frac{G\left(n_{1}\right)}{n_{\mathrm{eff}}}
$$




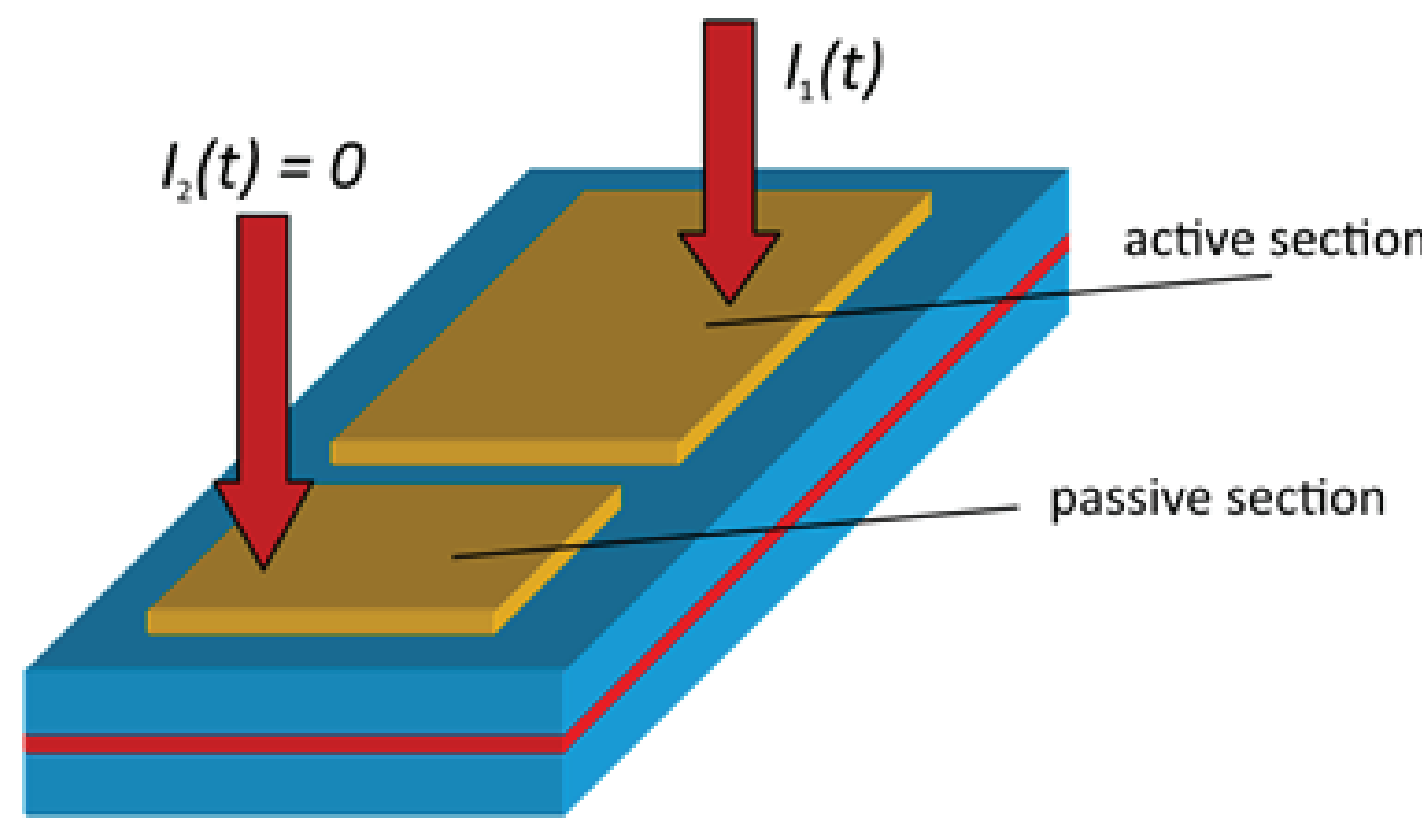

Figure 2: Schematic of a two-segment laser diode.

$$
\begin{gathered}
\frac{d n_{2}}{d t}=\eta_{i} \cdot \frac{I_{2}(t)}{q \cdot V q w_{2}}-\frac{n_{2}}{\tau_{s p}\left(n_{2}\right)}-c \cdot S \cdot \frac{G\left(n_{2}\right)}{n_{\mathrm{eff}}}+\frac{n_{1} e^{\left(-\sigma\left(n_{2}\right) \cdot L_{2}\right)} \cdot k_{s p 2}}{\tau_{s p}\left(n_{1}\right)} \cdot \frac{\gamma_{1}}{\gamma_{2}} \\
\frac{d S}{d t}=\left(\frac{c}{n_{\mathrm{eff}}} \cdot G\left(n_{1}\right) \cdot \gamma-\frac{1}{\tau_{p h 1}(t)}\right) \cdot S \cdot \gamma_{1}+\left(\frac{c}{n_{\mathrm{eff}}} \cdot G\left(n_{2}\right) \cdot \gamma-\frac{1}{\tau_{p h 2}(t)}\right) \\
\cdot S \cdot \gamma_{2}+\beta_{s p} \cdot \frac{n_{1}}{\tau_{s p}\left(n_{1}\right)} \cdot \gamma \cdot \gamma_{1}+\beta_{s p} \cdot \frac{n_{2}}{\tau_{s p}\left(n_{2}\right)} \cdot \gamma \cdot \gamma_{2}
\end{gathered}
$$

In equations (1-3), $G$ - modal gain; $\sigma$ - optical absorption coefficient; $S$ - photon density in the cavity; $k_{s p 1}$ and $k_{s p 2}$ are the coupling coefficients that describe the portion of the spontaneous emission from one section absorbed in the other; $n_{1}$ - free carrier density in the active section; $n_{2}$ - free carrier density in the passive section; $\tau_{s p}$ effective carrier lifetime; $\beta_{s p}$-spontaneous emission factor; $q$ - electron charge; $\tau_{p h}$ - photon lifetime for the lasing mode; $n_{\text {eff }}$ - effective refractive index for a given mode structure, $c$ - speed of light in a vacuum, $\gamma$-optical confinement factor of the laser active region; $\gamma_{1}, \gamma_{2}$-optical confinement factors for the active and passive sections, respectively.

Experimental gain versus current density dependence obtained by analyzing the differential efficiency versus cavity length dependence was used in simulations.

To approximate gain dependence on the carrier density we used equations (4-5):

$$
g(n)=\frac{\left(3.489 * 10^{17} * n-100\right)}{\Gamma} \text { if } n<2.827 * 10^{18} \mathrm{~cm}^{-3}
$$




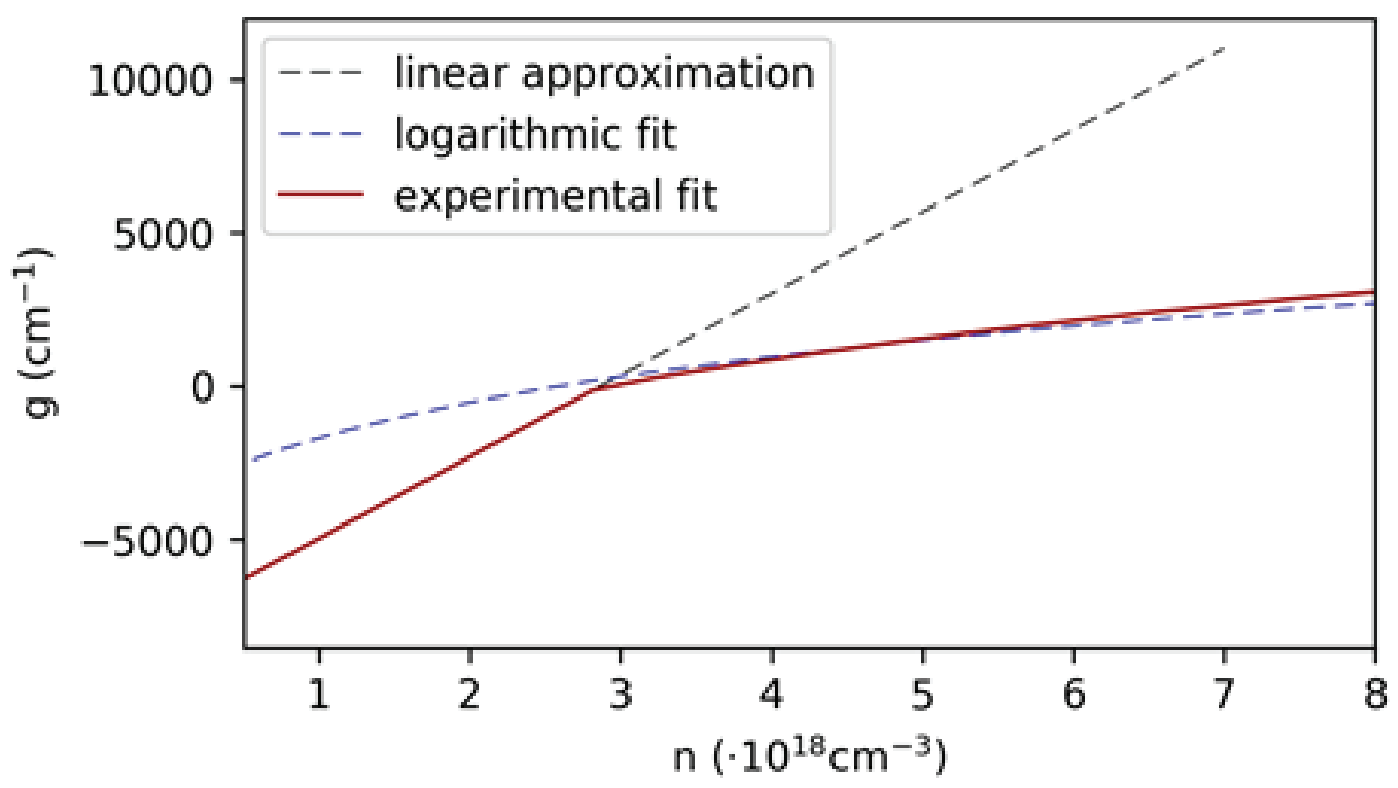

Figure 3: Experimental gain versus carrier density curve.

$$
g(n)=\frac{-2285.18+53.213 * \ln \left(n+1.546 * 10^{18}\right)}{\Gamma} \text { if } n \geq 2.827 * 10^{18} \mathrm{~cm}^{-3}
$$

In equations (4-5), $\mathrm{g}$ - material gain $\left(\mathrm{cm}^{-1}\right) ; n$ - free carrier density $\left(\mathrm{cm}^{-3}\right) ; \Gamma=$ 0.0131 - laser diode optical confinement factor. The epitaxial structure of the laser part comprised of a GaAs quantum well, $1 \mu \mathrm{m} \mathrm{Al} \mathrm{N}_{0.4} \mathrm{Ga}_{0.6}$ As undoped waveguide and two heavily doped $\mathrm{Al}_{0.6} \mathrm{Ga}_{0.4}$ As cladding layers. Net optical loss in this structure calculated via characterization in continuous-wave (CW) mode was estimated to be $0.3-0.5 \mathrm{~cm}^{-1}$.

To describe the pump current pulse, we used the results obtained in [10] for calculating the shape of the current pulse generated in a laser-thyristor. As was shown in that article, using this device it is possible to obtain a pulse of $2 \mathrm{~ns}$ duration and an amplitude of $15 \mathrm{~A}$. This can be achieved with a phototransistor, comprised of an $\mathrm{n}-\mathrm{Al}_{0.35} \mathrm{Ga}_{0.65} \mathrm{As}$ collector, lightly doped p-GaAs base, and n- $\mathrm{Al}_{0.15} \mathrm{Ga}_{0.85} \mathrm{As}$ emitter. It is well-known that when the laser is abruptly turned on, relaxation oscillations of the output power can be observed. They are caused by the difference between photon and carrier lifetimes. In the case of semiconductor lasers, the frequency of these oscillations lies in the $\mathrm{GHz}$ range. This means they can be observed during pulsed operation when the pulse length is about $1 \mathrm{~ns}$.

This effect can be seen in Figure 4, where the results of calculations for a standard single-section model are presented. Calculations were performed for a high-power laser with a cavity length of $3000 \mu \mathrm{m}$ and a stripe width of $200 \mu \mathrm{m}$. When pumping such a laser with a 10 A current pulse, only the first relaxation peak with a duration about 
of 60 ps is clearly seen. Such a result corresponds qualitatively to what is observed in practice.

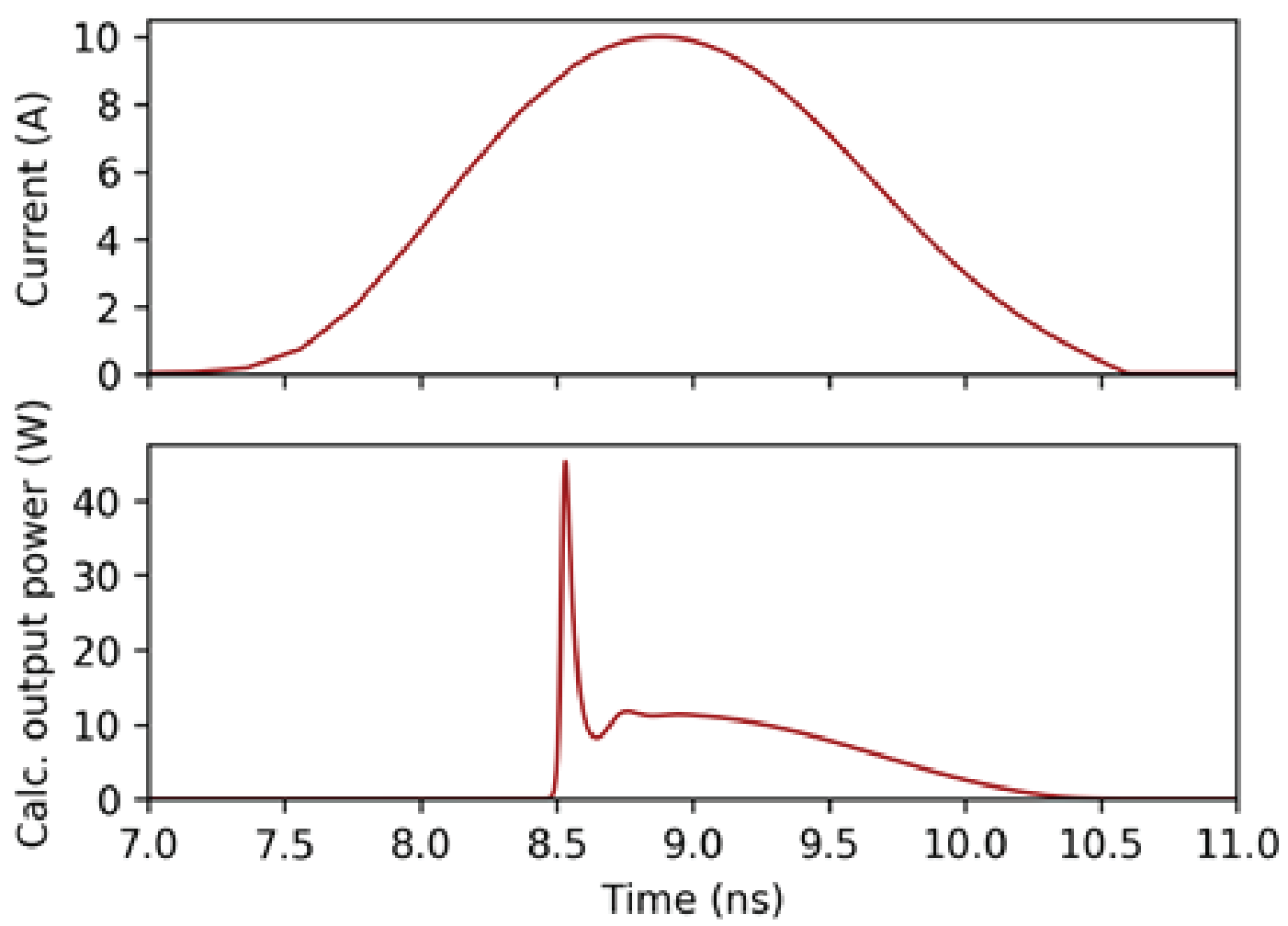

Figure 4: Current and output power dynamics of a single-section semiconductor laser. Resonator length $L$ $=3000 \mu \mathrm{m}$, stripe width $W=200 \mu \mathrm{m}$.

\section{Simulation Results}

Figures 5 and 6 show the simulation results for a two-section laser with a fixed cavity length $(3000 \mu \mathrm{m})$ and variable length of the passive section (o to $900 \mu \mathrm{m})$. Lateral guiding is implemented via a 200- $\mu m$-wide deep-ridge waveguide. It can be seen that adding a passive section leads to an increase of the turn-on-delay. Thus, when choosing the optimal length of the passive section, it is possible to achieve almost complete suppression of the lasing after the first pulse. It can be seen that the dependence of the first oscillation peak power on the length of the passive section has a clear maximum. In this case, the energy of the first laser pulse corresponds to only 50\% of the total energy of the optical output. Optimal result ( $~ 80 \%$ of output power, 100 W peak power) is obtained when the passive section length is $900 \mu \mathrm{m}$. When the fraction of the passive section is further increased there is not enough time for the 
carrier density to reach threshold during the current pulse. Because of that the pulse peak power abruptly declines.

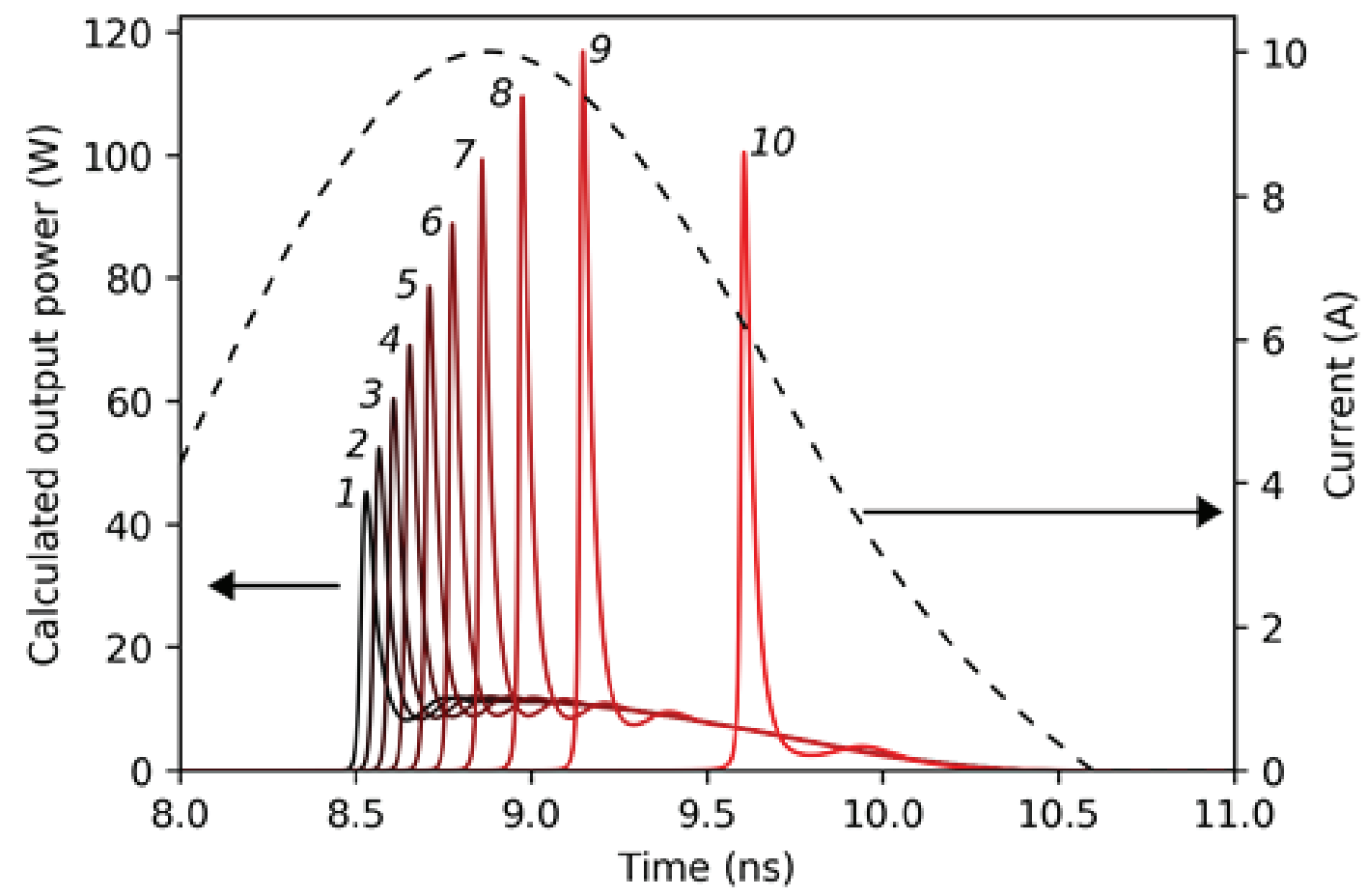

Figure 5: Calculated output power for different passive section lengths: $1-0 \mu \mathrm{m} ; 2-100 \mu \mathrm{m} ; 3-200 \mu \mathrm{m}$; 4-300 $\mu \mathrm{m} ; 5-400 \mu \mathrm{m} ; 6-500 \mu \mathrm{m} ; 7-600 \mu \mathrm{m} ; 8-700 \mu \mathrm{m} ; 9-800 \mu \mathrm{m} ; 10-900 \mu \mathrm{m}$. Resonator length $L=3000 \mu \mathrm{m}$, stripe width $W=200 \mu \mathrm{m}$.

Figure 7 shows time dependences of the free charge carrier density in the active and passive sections. It can be seen that in the active-section carrier dependence $n_{1}(t)$ has a shape that is not very different from the one normally observed in a conventional laser diode. There are several parts of the density dynamics curve: a part during which density rises to values above the stationary lasing threshold, a part during which density decreases when lasing turns on (which occurs during the first oscillation) and a part during which density reaches a steady-state value corresponding to the steadystate threshold current. In the passive section, the density dynamics is determined by the photon dynamics $S(t)$. In this case, even before lasing is turned on, there is a sharp increase in density in the passive section due to the same sharp increase of the active section emission. The density maximum value is reached when lasing is turned on and this value is close to the transparency density. It can be seen that when the first relaxation peak contains a major part of the whole laser pulse energy the laser pulse would have an optimal shape if the first oscillation corresponds to the decay of the current pulse. In this case, the current pulse does not provide the carrier density 


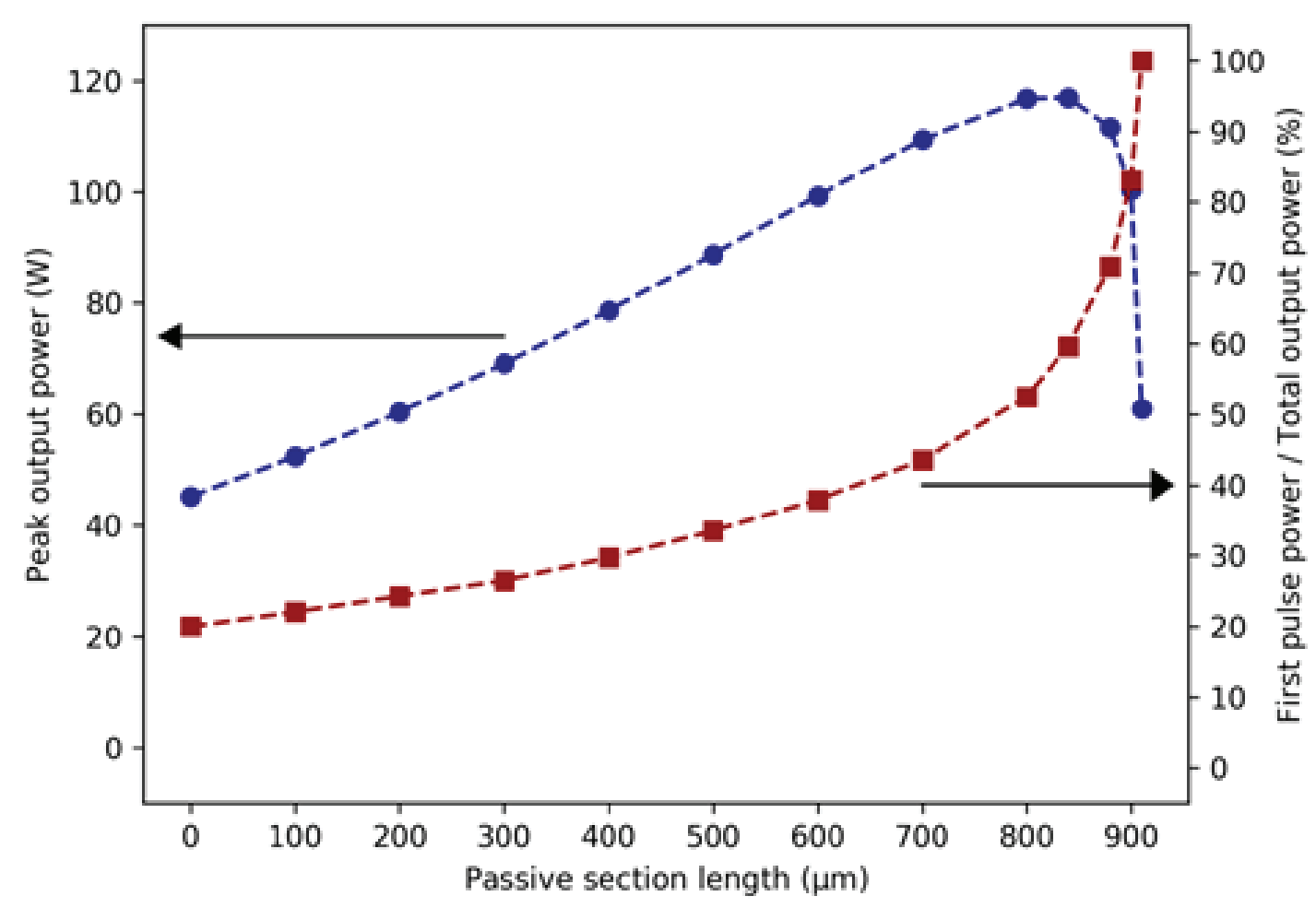

Figure 6: Calculated curves of peak output power and first pulse relative power vs passive section length. Resonator length $L=3000 \mu \mathrm{m}$, passive section length $L_{2}=3000 \mu \mathrm{m}$, stripe width $W=200 \mu \mathrm{m}$.

restoration in the active region after a sharp decrease below the threshold value during the first oscillation.

\section{Conclusion}

We have shown that by the means of a laser-thyristor it is both possible to achieve effective generation of current pulses in the ns range and laser pulses in the sub-ns range. It is shown that using a two-section structure allows to increase the efficiency of sub-ns lasing in laser-thyristors. In this case the size of the passive section significantly influences the dynamics of the generated laser pulses. It is found that the optimal length of the passive section providing the maximum portion of energy in the first oscillation is close to the maximum length for which there is no lasing for a given pump current pulse duration.

\section{References}

[1] Lanz, B., Ryvkin, B. S., Avrutin, E. A., et al. (2013). Performance improvement by a saturable absorber in gain-switched asymmetric-waveguide laser diodes. Optics 


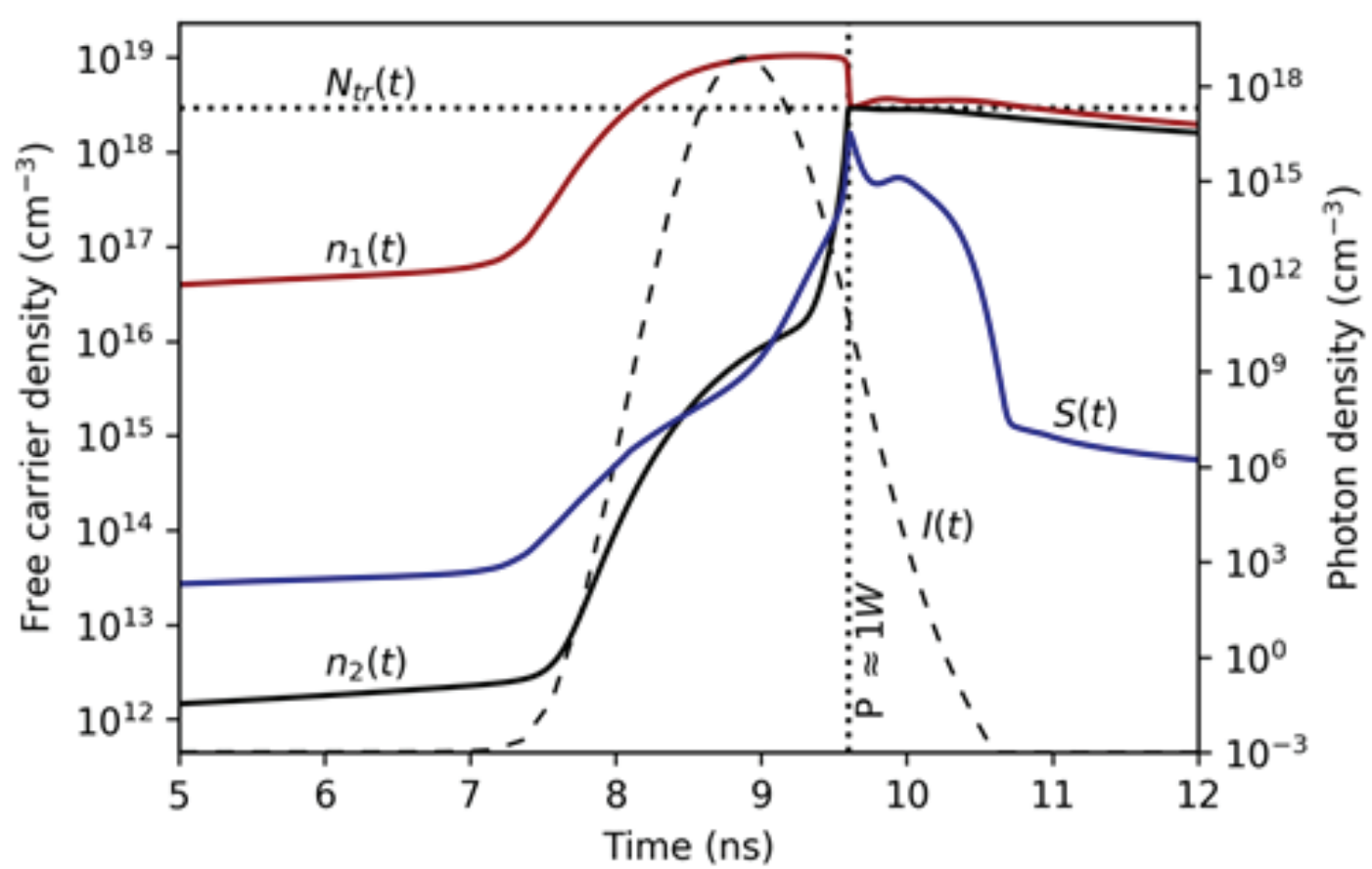

Figure 7: Calculated time dependencies of free carrier densities in the active $\left(n_{1}\right)$ and the passive $\left(n_{2}\right)$ section and the photon density $(S)$. Resonator length $L=3000 \mu \mathrm{m}$, passive section length $L_{2}=3000 \mu \mathrm{m}$, stripe width $W=200 \mu \mathrm{m}$. Pulse current (dashed line), transparency carrier density $N_{t r}$ (dotted line) and turn-on time (dotted line) are provided for reference.

Express, vol. 21, pp. 29780-29791.

[2] Hallman, L. W., Haring, K., Toikkanen, L., et al. (2012). 3 nJ, 100 ps laser pulses generated with an asymmetric waveguide laser diode for a single-photon avalanche diode time-of-flight (SPAD TOF) rangefinder application. Measurement science and Technology, vol. 23, pp. 025202-1-025202-8.

[3] Lanz, B., Vainshtein, S. N., and Kostamovaara, J. T. (2006). High power gain-switched laser diode using a superfast GaAs avalanche transistor for pumping. Applied Physics Letters, vol. 89, pp. 081122-1-081122-3.

[4] Podoskin, A. A., Soboleva, O. S., Zakharov, M. S., et al. (2015). Optical feedback in 905 nm power laser-thyristors based on AlGaAs/GaAs heterostructures. Semiconductor Science and Technology, vol. 30, pp. 125011-1-125011-6.

[5] Slipchenko, S. O., Podoskin, A. A., Rozhkov, A. V., et al. (2013). High-power pulse semiconductor laser-thyristor emitting at 900-nm wavelength. IEEE Photonics Technology Letters, vol. 25, pp.1664-1667.

[6] Podoskin, A. A., Soboleva, O. S., Zolotarev, V. V., et al. (2016). Laser-thyristors as a source of high-power laser pulses with a pulse width of 1-100 ns. IEEE Laser Optics (LO) 2016 International Conference, 7549719. 
[7] Ripper, J. E. and Dyment, J. C. (1968). Internal Q switching in GaAs junction lasers. Applied Physics Letters, vol. 12, pp. 365-367.

[8] Lau, K. Y. (1988). Gain switching of semiconductor injection lasers. Applied Physics Letters, vol. 52, pp. 257-259.

[9] Slipchenko, S. O., Yuferev, V. S., Podoskin, A. A., et al. (2015). Specific features of the injection processes dynamics in high-power laser thyristor. IEEE Transactions on Electron Devices, vol. 62, pp. 4091-4096.

[10] Slipchenko, S. O., Podoskin, A. A., Soboleva, O. S., et al. (March 4, 2016). Dynamic model of pulsed laser generators based on multi-junction N-p-N- i-P heterostructures. SPIE 9742, Physics and Simulation of Optoelectronic Devices XXIV, 97420l. 